\title{
Predicting Short-term Eurodollar Futures
}

\begin{abstract}
We propose and illustrate a structural model for the forward curve produced by Eurodollar futures contracts. Our model provides a three-part functional decomposition of the forward rate: a long-term, unconditional component, a maturity-specific component, and a date-specific component. The maturityspecific component captures preferred investment habitats, and the date-specific component captures shocks to expectations of future spot rates. These functional components (modeled with exponential basis functions) of the decomposition aggregate to an arbitrage-free representation of the underlying stochastic process that drives the evolution of the Eurodollar forward curve. We demonstrate the use of this approach by fitting this model to yields over the period $12 / 9 / 1981$ to $1 / 28 / 2008$. The estimation is accomplished by using a Kalman filter to determine the underlying representation. The estimated yield curve provides better out-of-sample predictions than the standard random walk model in forecasts over various horizons. We further show the profitability of a trading scheme that chooses futures positions based upon the anticipated forward curve.
\end{abstract}

JEL Classification number: C53, E43, E47.

Keywords: Term Structure, Interest Rates, Forward Rates, Forecasting

CHOONG TZE CHUA is an Assistant Professor of Finance at Singapore Management University, Singapore. ctchua@smu.edu.sg

KRISHNA RAMASWAMY is Edward Hopkinson, Jr Professor of Finance at The Wharton School of the University of Pennsylvania, Philadelphia, PA. krishna@wharton.upenn.edu

ROBERT A. STINE is a Professor of Statistics at The Wharton School of the University of Pennsylvania, Philadelphia, PA. stine@wharton.upenn.edu 


\begin{abstract}
We propose and illustrate a structural model for the forward curve produced by Eurodollar futures contracts. Our model provides a three-part functional decomposition of the forward rate: a long-term, unconditional component, a maturity-specific component, and a date-specific component. The maturityspecific component captures preferred investment habitats, and the date-specific component captures shocks to expectations of future spot rates. These functional components (modeled with exponential basis functions) of the decomposition aggregate to an arbitrage-free representation of the underlying stochastic process that drives the evolution of the Eurodollar forward curve. We demonstrate the use of this approach by fitting this model to yields over the period $12 / 9 / 1981$ to $1 / 28 / 2008$. The estimation is accomplished by using a Kalman filter to determine the underlying representation. The estimated yield curve provides better out-of-sample predictions than the standard random walk model in forecasts over various horizons. We further show the profitability of a trading scheme that chooses futures positions based upon the anticipated forward curve.
\end{abstract}


Essentially, all models are wrong, but some are useful. — George E.P. Box

Structural models of the dynamics of interest rates have many applications. They play an important role in the design and management of fixed income portfolios and in the valuation and hedging of more complex securities. An analyst of the fixed-income market can choose from among several alternative models, such as the Vasicek or the Cox, Ingersoll and Ross (CIR) models. An important characteristic that guides the choice from among these alternatives is the ability of the model to forecast forward rates or futures prices.

In a recent paper (Chua et al [2008], henceforth CFRS) the authors proposed a general class of affine, arbitrage-free models that are then fit to the current term structure of interest rates. Each member of this class of models corresponds to a representation of an underlying stochastic process which can be estimated through a parsimonious set of state variables. As a demonstration, CFRS empirically select and estimate a member of this class using a sample of observed "training" data. The resulting model gave good out-of-sample forecasts of US Treasury yields when compared to several extant models that include the random walk model.

Each member of this CFRS class of arbitrage-free models represents the forward curve on any date as the sum of three curves:

1. An unconditional curve that represents the steady-state forward curve;

2. A maturity-specific curve that embeds the influence of supply and demand from agents who have needs for loans of specific terms; and

3. A date-specific curve that embeds expectations about spot rates to prevail at fixed future dates.

The maturity-specific curve describes deviations from the long-run, unconditional curve due to the behavior of investors who have preferences for specific investment horizons or to a preferred habitat model (see for example Modigliani \& Sutch [1966]). Because the curve of maturity-specific deviations from the long-term unconditional curve embeds investors' maturity preferences, expectations of future spot rates play no role in this component. The date-specific curve embeds all of the information that investors have regarding the levels of future spot rates and summarizes the effects of fundamental monetary influences on expected future interest rates. The maturity-specific and date-specific curves connect to well-established financial models. The curve of maturity-specific deviations corresponds to the deviations from the steady state curve in extant equilibrium models of interest rates (such as Cox, Ingersoll and Ross [1985] or Vasicek [1977]). The curve of date-specific deviations corresponds to the influences of the expectations hypothesis. In this sense, the fitted parametric model exhibited in CFRS [2008] combines both investors' maturity-preferences and their current expectations. The model has the additional advantage of being demonstrably arbitrage-free. 
Our objective here is to show that a representative member chosen from this class of models is able forecast 90-day forward rates to prevail at future dates. In the actual estimation we use Eurodollar futures contracts to impute the forward rates. ${ }^{1}$ We first select a member of the CFRS class using the Akaike information criterion over an initial set of training data; these data are also used to estimate the parameters of the chosen model. We then roll this model forward and compare the accuracy of its forecasts with the standard random walk over a subsequent hold-out sample. We also report on the profitability and risk of a strategy that trades the Eurodollar futures using forecasts produced by our model. Our results indicate that the model is useful, most notably at longer forecast horizons.

\section{A BRIEF DECSRIPTION OF THE MODEL}

The model for the forward curve at date $t$ is written $F(\tau ; t)$ and represents the curve of forward rates for instantaneous loans to begin at future dates $t+\tau, \tau>0$. The first argument $\tau$ refers to the time to maturity; the second argument $t$ refers to the calendar date for that curve. Our proposed model of the forward curve decomposes $F(\tau ; t)$ as the sum of three component curves:

$$
F(\tau ; t)=U(\tau)+M(\tau ; t)+D(\tau ; t)
$$

where

1. $U(\tau)$ is the unconditional or steady-state forward curve;

2. $M(\tau ; t)$ is the maturity-specific curve for deviations from $U(\tau)$; and

3. $D(\tau ; t)$ is the date-specific curve for deviations from $U(\tau)$.

In keeping with our notation for the forward curve, $M(\tau ; t)$ refers to the maturity-specific deviation embedded in the forward curve at date $t$ for the future date $t+\tau$.

The function $U(\tau)$ represents the steady state or the unconditional forward curve. If we were to forecast the forward curve at a time in the very distant future, all presently available information would be of little use. Let $E_{t}$ represent the expectation operator given the set of all information available up to time $t$. This unconditional curve can be written:

$$
U(\tau)=\lim _{s \uparrow \infty} E_{\mathrm{t}}[\mathrm{F}(\tau ; s)]
$$

The unconditional curve is time invariant and may be estimated by taking an average of all available historical curves. 
The concept of a maturity-specific effect originates from the Market Segmentation Hypothesis and the Preferred Habitat Theory (Modigliani \& Sutch [1966]). That model postulates that some market participants are primarily concerned with their natural maturity habitat, with little regard for the implication of the forward rates on future spot rates. The maturity-specific deviation $M(\tau ; t)$ in Equation (1) captures that effect. That deviation is localized to particular maturities of the forward curve. The actions of participants with preferred habitats affect only those maturities (and nearby maturities) of the forward curve rather than move progressively towards shorter maturities and eventually affect the spot rates. Therefore, $M(\tau ; t)$ captures abnormal activity that affects the forward curve at specific maturities $\tau$. We model the maturity-specific curve as a point-wise mean-reverting process that reverts to zero at a constant rate, so that

$$
E_{t}[M(\tau ; T)]=e^{-K_{m}(T-t)} M(\tau ; t) \quad \tau>0
$$

where the parameter $K_{m}>0$ determines the speed of reversion to zero. The overall maturity-specific curve can be comprised of two or more maturity-specific deviations, for example,

$$
M(\tau ; t)=M_{1}(\tau ; t)+M_{2}(\tau ; t)
$$

The components $M_{1}(\tau ; t)$ and $M_{2}(\tau ; t)$ may mean-revert to zero at different rates. Thus, for each component of the maturity-specific deviation, we require that

$$
E_{t}\left[M_{j}(\tau ; T)\right]=e^{-K_{m_{j}}(T-t)} M_{j}(\tau ; t) \quad \tau>0, j=1,2, \ldots
$$

The arbitrage-free formulation of the overall curve of maturity-specific deviations has the property that $M(\infty ; t)=0$ for all $t$. Note that instantaneous or spot rates are zero maturity loans, and we assume $M(0 ; t)=0$ for all $t$ to allow the date-specific deviations to capture the dynamics of present and future spot rates. Exhibit 1 illustrates the forecasted behavior of maturity-specific deviations. The curve is anchored at zero at extreme maturity values, and the entire curve decays (in expectation) point-wise towards zero as time passes, satisfying Equation (3).

\section{**** EXHIBIT 1 AROUND HERE $* * * *$}


In contrast, the date-specific curve represents information that affects the expectation of the spot interest rate to prevail on a specific calendar date in the future. The concept of a date-specific deviation has its roots from the Expectations Hypothesis (Fisher [1896]). It is intuitive that forward rates observable rates at which one can lock in borrowing and lending at future dates - contain information regarding future spot rates. Therefore a high forward rate today should naturally point towards a higher spot rate at the corresponding date in the future. However, the Expectations Hypothesis fails in some basic ways, as shown in the literature. For instance, in the theoretical realm, Cox, Ingersoll and Ross [1981] show that some versions of the Expectations Hypothesis admit arbitrage. ${ }^{2}$ In empirical tests, forecasts of forward rates generated by the Expectations Hypothesis model are generally inferior to even the most basic benchmark, the random walk. The model proposed here attributes only a part of the current forward curve as containing information about future spot rates.

The date-specific deviation curve $D(\tau ; t)$ is influenced by abnormal events or information that affects the portions of the forward curve corresponding to specific maturity dates. In other words, this curve captures the deviations of expected future spot rates from the unconditional spot rate. For instance, suppose that on $t \equiv$ January 12008 it is learned that the Treasury needs new additional financing on (or around) $s \equiv$ January 2009. This borrowing will drive up interest rates during that period. On January 1 2008, the 1-year forward rate would be elevated. As time passes, we expect the elevated portion of the forward curve to move closer to the origin since in expectation the higher rates around January 12009 would remain. Thus, the date-specific deviation has the property:

$$
E_{t}[D(s-T ; T)]=D(s-t ; t) \quad \text { for } t<T<s
$$

The date-specific deviation at zero maturity is simply the difference between the spot interest rate and the unconditional spot rate: $D(0 ; t)=F(0 ; t)-U(0)$. At infinite maturity, the date-specific deviation must be zero because it is not plausible that one can have any information about the spot rate in the infinite future other than that contained in the unconditional spot rate. Hence, $D(\infty ; t)=0$ for all $t$. Exhibit 2 illustrates the forecasted behavior of the date-specific curve. Starting from a given date-specific curve that is anchored at zero at the long end, the entire curve shifts (in expectation) to the left as time passes, satisfying Equation (5).

\section{**** EXHIBIT 2 AROUND HERE ****}




\section{The Dynamic Behavior of the Forward Curve}

The dynamic behavior of the forward curve in Equation (1) depends on the dynamic behavior of the date-specific and the maturity-specific curves, as indicated in Equations (3) and (5). Each of these, within a specific model that we specify in the next section, is affected by one or more state variables that represent the evolution of underlying economic factors.

The maturity-specific deviation is caused by abnormal pricing of forward rates specific to certain maturities, driven by habitat and preferences of individual and institutional investors. Changes in demand or supply at a given maturity habitat can affect a range of surrounding maturities — investors treat them as close substitutes - which allows us to treat the maturity-specific deviation as a smooth curve. Since these are deviations from the average, the average deviation should naturally be zero. Without additional information to guide us on how these deviations behave over time, a simple yet intuitive model for these deviations would be that they decay towards zero at some rate. In the next section, where we develop an arbitrage-free framework for our model, we assume that the maturity-specific deviation decays at an exponential rate to satisfy the Heath-Jarrow-Morton requirement for the model to be arbitrage-free.

Our model (in the general form under discussion so far) does not a priori preclude the possibility that there might be negative forward rates. Given an observed term structure of forward rates that is positive at all maturities, it is possible to find maturity-specific and date-specific deviations that fit the current term structure but produce forecasts of negative forward rates in the future. For example, an extremely large and positive maturity-specific deviation coupled with an extremely large and negative date-specific deviation can produce such negative forward rate forecasts. However, in the explicit parameterized forms of the model described in the section below we ensure that the model is arbitrage-free by checking the HJM restrictions.

In the implementations of explicit forms of our general model we employ sums of exponential basis functions for $U(\tau)$ and similar basis functions (that are scaled by Brownian motions) to specify dynamic functional forms for $M(\tau ; t)$, and $D(\tau ; t)$. The resulting model for forward rates $F(\tau ; t)$ is exponentially affine in the state variables and has a structure that lends itself to estimation. ${ }^{3}$

\section{A ONE-FACTOR ILLUSTRATION OF THE MODEL}

As a first step, it is useful to consider a basic dynamic model for the forward rate that is driven by a single Brownian motion — we denote this $F_{1}(\tau ; t)$ with the subscript " 1 " indicating the number of Brownian motions. ${ }^{4}$ In this basic setting, the random variation that affects the maturity-specific curve is perfectly correlated with the random variation that drives the date-specific curve. The explicit 
parameterization is chosen as a linear combination of exponential basis functions. ${ }^{5}$ The three components of the current forward curve $F_{1}(\tau ; t)$ are as follows:

1. The time-invariant unconditional curve is now explicitly written as

$U_{1}(\tau)=C_{0}-C_{1} e^{-2 K_{m} \tau}$

where $C_{0}, C_{1}$ and $K_{m}$ are positive constants to be estimated from the data. This form generates a smooth upward-sloping unconditional curve that starts at $U_{1}(0)=C_{0}-C_{1}$ at the origin and asymptotes to $C_{0}$ at infinite maturity.

2. The maturity-specific deviation is explicitly written as

$M_{1}(\tau ; t)=m(t)\left[e^{-K_{m} \tau}-e^{-2 K_{m} \tau}\right]$

By design, $M_{1}(0 ; t)=0$ for all $t$. Because $\lim _{\tau \rightarrow \infty} M(\tau ; t)=0$ the deviation has a humped shape with a peak at maturity $\tau=\frac{\ln 2}{K_{m}}$. The stochastic process $m(t)$ is an Itô process whose dynamics are induced by the Brownian motion, defined further below; $m(t)$ serves to scale the deviation which has a fixed shape.

3. The date-specific deviation is specified as

$$
D_{1}(\tau ; t)=d(t)\left[e^{-2 K_{m} \tau}\right]
$$

Here $d(t)$ is an Itô process whose dynamics are related to the Brownian motion, also defined below; it serves to scale an exponential function which is either monotonically upward- or downward-sloping. Note that the overall date-specific deviation $D_{1}(0 ; t)=d(t)$ at zero maturity, and it asymptotes to zero at infinite maturity $\left(D_{1}(\infty ; t)=0\right)$, reflecting the fact that there can be no expectation about the spot rate in the distant future other than the long-run mean.

Given this parameterization, Itô's lemma implies that the model forward rate obeys the following SDE:

$$
\mathrm{d} F_{1}(\tau ; t)=\frac{\partial F_{1}}{\partial t} \mathrm{~d} t+\frac{\partial F_{1}}{\partial m(t)} \mathrm{d} m(t)+\frac{\partial F_{1}}{\partial d(t)} \mathrm{d} d(t)
$$


indicating dependence on the driving Itô processes $m(t)$ and $d(t)$; all second-order terms are zero.

Recall that the model requires (see Equation (3)) the maturity-specific deviation to decay exponentially towards zero at rate $K_{m}$. Therefore we require the SDE for the state variable $m(t)$ to have the drift $-m(t) K_{m}$, and specify its diffusion coefficient $\gamma_{t}$ later (when we impose the arbitrage-free condition):

$$
\mathrm{d} m(t)=-m(t) K_{m} \mathrm{~d} t+\gamma_{t} \mathrm{~d} B(t)
$$

where $B(t)$ is the one Brownian motion for this parameterization.

In the SDE for the Itô process $d(t)$ we make its drift rate equal to $-2 d(t) K_{m}$ so that we satisfy the Equation (5) above. We specify the diffusion of the process $d(t)$ to be identical to that of $m(t)$ in order to ensure that the drift and diffusion of the forward rate conform to the HJM condition:

$$
\mathrm{d} d(t)=-2 d(t) K_{m} \mathrm{~d} t+\gamma_{t} \mathrm{~d} B(t)
$$

Note that the maturity-specific curve and the date-specific curve are driven by the same Brownian motion, so that their innovations are perfectly correlated. By choosing the overall forward curve as the sum of several components driven by multiple Brownian motions (as we do below) we avoid this extreme implication.

Equation (9), the SDE for the forward rate in this explicit 1-Brownian motion setup can now be rewritten as:

$$
\mathrm{d} F_{1}(\tau ; t)=\left\{-K_{m}\left(2 C_{1}+m(t)\right) e^{-2 K_{m} \tau}\right\} \mathrm{d} t+\left\{e^{-K_{m} \tau} \gamma_{t}\right\} \mathrm{d} B t
$$

The appendix details the proof that this model conforms to HJM's specifications for no-arbitrage. The pricing of bonds as well as interest rate derivatives are also quite straightforward within the context of this model (see CFRS [2008] for more details).

\section{THE FORECASTING VERSION OF THE MODEL}

Before we describe the version of the model we chose for building forecasts, it is useful to describe the data we use. 


\section{Data: Eurodollar Futures}

For the period 12/9/1981 to 1/28/2008, we obtain daily prices of all Eurodollar futures contracts listed in the Chicago Mercantile Exchange.

The Eurodollar futures price on date $t$ for maturity on date $t+\tau, P(\tau ; t)$, refers to 100 minus the annualized 90-day Libor rate for the period $t+\tau$ to $t+\tau+90$. Since the CFRS [2008] model is built around instantaneous forward rates, we make the simplifying assumption that the instantaneous forward rate in the middle of the 90-day period referenced by the Eurodollar futures contract equals the annualized forward rate implied by the Eurodollar futures price as the standard annualized discount rate: $f(\tau+45 ; t)=$ $100-P(\tau ; t)$.

Summary statistics of the implied forward rates are displayed in Exhibit 3. A typical day would see approximately 40 active contracts, with the maximum being 45 . The maturities of the forward rates range from 45 days (corresponding to a Eurodollar futures contract that expires at the end of that trading day) to slightly more than 10 years. The training sample, which is an early sub-sample of the data, is more sparsely populated with an average of (approximately) 10 active contracts per trading day, with maturities stretching out to 4 years.

\section{**** EXHIBIT 3 AROUND HERE $* * * *$}

One feature of the data that merits special attention is what appears to be a microstructure effect: there are persistent blips in the data for December contracts, perhaps caused by those who use these contracts for swaps. These blips occur systematically and our model - whose form and whose dynamics are both smooth - cannot accommodate them. In order to build a useful model for prediction, we elected to incorporate these microstructure features into the forecast. We describe this adjustment used in the section titled Generating Model Forecasts.

\section{Model Selection using the AIC Criterion}

In this section we describe the model we chose (from alternative parameterizations) and briefly describe the procedure employed in that choice.

We restricted the alternative models to have no more than 4 Brownian Motions (BMs) that serve as driving state variables. The advantage of our general model-building procedure is that we can bifurcate the influence of each BM to impact the maturity-specific deviation, or the date-specific deviation, or both. In 
this way we can permit the shape of the forward curve to accommodate several humps and also allow the dynamics of the forward curve to be influenced by correlated deviations driven by independent BMs.

To choose from the list of alternative models, we fit each model over the period 12/09/1981 to 10/28/1991, using daily data from the Eurodollar futures market (2500 days of data). Each estimation employs the Kalman filter in the manner explained in next section. The various alternative models are generated by combining different sub-models (also referred to as arbitrage-free units or AFU, in CFRS [2008]). CFRS [2008] also details the proof that combinations of these sub-models are arbitrage-free.

Because the alternative parameterizations involve forms with varying numbers of parameters, we used the Akaike information criterion (henceforth AIC, see Akaike [1973]) to choose among the models. Exhibit 4 shows the descriptions of the various candidate models and their respective AIC numbers. Model 5 obtains the lowest AIC among the 6 candidate models. We therefore chose Model 5 (henceforth CFRS_ED model) for empirical implementation reported in the rest of this paper.

**** EXHIBIT 4 AROUND HERE $* * * *$

The CFRS_ED model, driven by 3 independent Brownian motions, is the sum of the unconditional curve, 2 maturity-specific curves, and 4 date-specific curves fitted to 3 exponential basis functions $\left\{\mathrm{e}^{-K_{m}}, e^{-2 K_{m}}, e^{-4 K_{m}}\right\}$. We denote the error term in the fitted model as $\varepsilon(\tau ; t)$ :

$f(\tau ; t)=F_{3}(\tau ; t)+\varepsilon(\tau ; t)$

so that

$f(\tau ; t)=\left(e^{\vec{k} \tau}\right)^{\prime}(\vec{u}+\mathbf{M} \vec{m}(t)+\mathbf{D} \vec{d}(t))+\varepsilon(\tau ; t)$

where

$$
\left(e^{\vec{k} \tau}\right)=\left[\begin{array}{c}
1 \\
e^{-K_{m} \tau} \\
e^{-2 K_{m} \tau} \\
e^{-4 K_{m} \tau}
\end{array}\right], \mathbf{M}=\left[\begin{array}{cc}
0 & 0 \\
1 & 2 \\
-1 & -1 \\
0 & -1
\end{array}\right], \mathbf{D}=\left[\begin{array}{llll}
0 & 0 & 0 & 0 \\
0 & 0 & 0 & 0 \\
1 & 1 & 0 & 1 \\
0 & 0 & 1 & 0
\end{array}\right]
$$


$\vec{m}(t)=\left[\begin{array}{l}m_{1}(t) \\ m_{2}(t)\end{array}\right], \vec{d}(t)=\left[\begin{array}{l}d_{1}(t) \\ d_{2}(t) \\ d_{3}(t) \\ d_{4}(t)\end{array}\right], \vec{u}=\left[\begin{array}{c}C_{0} \\ 0 \\ -C_{1} \\ -C_{2}\end{array}\right]$

Therefore there are 6 state variables in this system: $m_{1}(t), m_{2}(t), d_{1}(t), d_{2}(t), d_{3}(t)$ and $d_{4}(t)$.

The stochastic processes for vectors $\vec{m}(t)$ and $\vec{d}(t)$ are:

$\mathrm{d} \vec{m}(t)=\mathbf{V}_{m} \vec{m} \mathrm{~d} t+\Sigma_{m}(\vec{m}) \mathrm{d} \vec{B}(t)$

$\mathrm{d} \vec{d}(t)=\mathbf{V}_{d} \vec{d} \mathrm{~d} t+\Sigma_{d}(\vec{m}) \mathrm{d} \vec{B}(t)$

where

$\mathbf{V}_{m}=\left[\begin{array}{cc}-K_{m} & 0 \\ 0 & -K_{m}\end{array}\right], \mathbf{V}_{d}=\left[\begin{array}{cccc}-2 K_{m} & 0 & 0 & 0 \\ 0 & -2 K_{m} & 0 & 0 \\ 0 & 0 & -4 K_{m} & 0 \\ 0 & 0 & 0 & -2 K_{m}\end{array}\right]$

$\Sigma_{m}(\vec{m})=\left[\begin{array}{ccc}\gamma_{1, t} & 0 & 0 \\ 0 & \gamma_{2, t} & 0\end{array}\right], \Sigma_{d}(\vec{m})=\left[\begin{array}{ccc}\gamma_{1, t} & 0 & 0 \\ 0 & \gamma_{2, t} & 0 \\ 0 & \gamma_{2, t} & 0 \\ 0 & 0 & \gamma_{3, t}\end{array}\right], d B_{t}=\left[\begin{array}{c}d B_{1, t} \\ d B_{2, t} \\ d B_{3, t}\end{array}\right]$

using 3 independent Brownian motions, and

$\gamma_{1, t}^{2}=\left(m_{1}(t)+C_{1}\right) K_{m}^{2}$

$\gamma_{2, t}^{2}=\left(m_{2}(t)+C_{1}\right) \frac{K_{m}^{2}}{4}$

$\gamma_{3, t}^{2}=\left(3 m_{2}(t)+4 C_{2}\right) 2 K_{m}^{2}$ 
Note that, in the model selection process, we limit the choice of models to those having at most $4 \mathrm{BMs}$ and a few alternative forms within that restriction; it is possible that more extensive forms may perform better than our model in forecast accuracy.

\section{Generating Model Forecasts}

Suppose that on date $T$, we want to forecast future forward rates for date $T_{2}$. We use a three-step process:

1. Over the parameter space of $\left\{K_{m}, \sigma^{* 2}\right\}$, (which are the rate of decay of the maturity-specific deviations and the variance of the measurement errors of the Kalman filter, respectively) search for a point that maximizes the quasi-likelihood. The quasi-likelihood for any point in the space is obtained via the following procedure:

(a) Use all the data across dates and maturities for the Eurodollar forward rates, which we now label as $f(\tau ; t)$, for all dates up to date $T$ to fit the unconditional forward curve of the form: $U(\tau)=C_{0}-C_{1} e^{-2 K_{m} \tau}-C_{2} e^{-4 K_{m} \tau}$

(b) Subtract the fitted unconditional forward curve from the observed forward rates to obtain the cross-section of deviations. These deviations form the "observations" in the context of the Kalman filter (The operations of the Kalman filter are detailed in the appendix): $\vec{z}_{t} \equiv f(\vec{\tau} ; t)-\hat{U}(\vec{\tau})=\mathbf{A} \vec{x}_{t}+\vec{\varepsilon}_{t}$

(c) Run the Kalman filter to estimate the state variables for the maturity-specific and date-specific curves.

(d) Determine the quasi-likelihood from the Kalman filter: $\ln L=-\frac{n T}{2} \ln 2 \pi-\frac{1}{2} \sum_{t=1}^{T}\left(\ln \left|H_{t}\right|+v_{t}^{\prime} H_{t}^{-1} v_{t}\right)$ where $H_{t}$ denotes the conditional covariance matrix of the prediction errors $v_{t}$. See equation (33) in the appendix.

2. Beginning with estimated state variables at date $T$ obtained in the previous step, we evolve these estimates forward according to their respective decay rates to obtain forecasts for future date $T_{2}$ :

$$
\begin{aligned}
& \hat{m}_{1}\left(T_{2}\right)=E\left[m_{1}\left(T_{2}\right) \mid \hat{m}_{1}(T)\right]=\hat{m}_{1}(T) e^{-\hat{K}_{m}\left(T_{2}-T\right)} \\
& \hat{m}_{2}\left(T_{2}\right)=E\left[m_{2}\left(T_{2}\right) \mid \hat{m}_{2}(T)\right]=\hat{m}_{2}(T) e^{-\hat{K}_{m}\left(T_{2}-T\right)} \\
& \hat{d}_{1}\left(T_{2}\right)=E\left[d_{1}\left(T_{2}\right) \mid \hat{d}_{1}(T)\right]=\hat{d}_{1}(T) e^{-2 \hat{K}_{m}\left(T_{2}-T\right)}
\end{aligned}
$$




$$
\begin{aligned}
& \hat{d}_{2}\left(T_{2}\right)=E\left[d_{2}\left(T_{2}\right) \mid \hat{d}_{2}(T)\right]=\hat{d}_{2}(T) e^{-2 \hat{K}_{m}\left(T_{2}-T\right)} \\
& \hat{d}_{3}\left(T_{2}\right)=E\left[d_{3}\left(T_{2}\right) \mid \hat{d}_{3}(T)\right]=\hat{d}_{3}(T) e^{-4 \hat{K}_{m}\left(T_{2}-T\right)} \\
& \hat{d}_{4}\left(T_{2}\right)=E\left[d_{4}\left(T_{2}\right) \mid \hat{d}_{4}(T)\right]=\hat{d}_{4}(T) e^{-2 \hat{K}_{m}\left(T_{2}-T\right)}
\end{aligned}
$$

3. Correct the model predictions to accommodate market microstructure effect (discussed above) present at time $T$. Exhibit 5 illustrates the type of effects captured by the adjustment described there. To that end, on the observed day $T$, we back out the forward curve using the current estimates of the underlying state variables, obtaining a fitted curve. We then subtract this fitted curve from the observed forward rates, producing $\varepsilon(\tau ; T)$. Because this microstructure persists in

time, our forecast for the future data is $\hat{f}\left(\tau ; T_{2}\right)=\left(e^{\vec{k} \tau}\right)^{\prime}\left(\hat{\vec{u}}+\mathbf{M} \hat{\vec{m}}\left(T_{2}\right)+\mathbf{D} \hat{\vec{d}}\left(T_{2}\right)\right)+\varepsilon\left(\tau+\left(T_{2}-T\right) ; T\right)$. In effect, we model the observed forward rates as a sum of the smooth curve implied by our model plus additive microstructure effects. Our assumption is that the imprecise cross-sectional fits are not measurement noise per se, which should dissipate over a longer term, but are market micro-structure distortions that can be expected to persist over the short-term.

\section{**** EXHIBIT 5 AROUND HERE ****}

\section{RESULTS}

In this section, we test the efficacy of our forecasts using 2 different yardsticks: (1) the accuracy of prediction of future forward rates implied by Eurodollar futures prices, as measured using RMSE; and (2) the profitability of a simple trading strategy uses the predictions of the model to generate trade signals.

It should be emphasized that the model selection uses only data up to 10/28/1991 while forecast generation, uses only the data that is available up to the date that the forecast is being made. This means that the forecasts made are truly out-of-sample. We make forecasts for 5-trading-days, 20-trading-days, 65-trading-days and 250-trading-days ahead, approximately corresponding to 1-calendar-week, 1calendar-month, 1-calendar-quarter and 1-calendar-year ahead, respectively. Refer to Exhibit 6 to get a graphical view of the model training and forecasting timeline.

**** EXHIBIT 6 AROUND HERE **** 


\section{Predictive Accuracy}

The forecast error are calculated as the differences between the forecasted forward rates and the actual Eurodollar futures implied forward rates that prevail at the end of the forecasting period. We compare the forecasts of the CFRS_ED model against the hypothesis that the Eurodollar futures prices of each contract remains unchanged over the forecasting period, the random walk model (RW model). For each date, we measure the cross-sectional square root of the mean squared forecast errors (RMSE). We report the timeseries average and standard deviation of these cross-sectional RMSEs in Panel A of Exhibit 7. We report the statistical significance of the differences between the RMSE of the CFRS_ED model and the RW model in Panel B of Exhibit 7.

The CFRS_ED model performs better than the random walk for all 4 forecast horizons, as evidenced by the lower forecast error RMSEs. The differences in RMSE start from 0.02 basis points (12.53 for the CFRS_ED model vs 12.55 for the RW model) for 5-day-ahead forecasts, and monotonically increase to an economically significant 6.31 basis points for 250-day-ahead forecasts (98.58 for the CFRS_ED model versus 104.89 for RW model). In terms of statistical significance, we calculate the Newey-West statistic (NW-stat; see Newey and West [1987]) for the differences between the RMSE of the CFRS_ED model and the RW model. ${ }^{6}$

The NW-stat takes into account serial correlation and heteroscedasticity in the time series of forecast errors; this structure is very evident in our situation because of the over-lapping forecasting windows. Panel B of Exhibit 7, shows that the CFRS_ED model is consistently more accurate, but the differences are not statistically significant at the usual levels.

\section{**** EXHIBIT 7 AROUND HERE ****}

\section{Trading Strategy Profitability}

We use the methodology described above to generate forecasts and signals to trade forward rates 5days, 20-days, 65-days, and 250-days ahead. Each trade signal generated is based on whether the forecasted forward rate is above or below the current Eurodollar-implied forward rate. If the forecasted rate is below the current rate, we go short that forward rate (equivalently, we go long the Eurodollar futures contract), and hold that short position until the end of the forecasting period. Conversely, if the forecasted rate is above the current rate, we go long the that forward rate (by going short the Eurodollar futures contract), and hold that long position until the end of the forecasting period. Trades are placed for 
all Eurodollar futures contracts that will still exist at the end of the forecasting period. The profitability on each trade is then calculated as the cross-sectional average movement in (or against) the predicted direction over the length of the forecasting period, expressed in basis points. For comparison, we also measure the profitability of an alternative trading strategy that always buys-and-holds a long position in all the traded Eurodollar futures contracts, which is equivalent to riding the yield curve. We report the means and standards deviations of the profitability of the CFRS_ED model trading strategy and the buyand hold strategy in Panel A of Exhibit 8. We report the statistical significance of the profitability of the CFRS_ED model trading strategy, as well as the statistical significance of the differences in profitability between the two strategies in Panel B of Exhibit 8.

In terms of profitability, the trading signals generated by the CFRS_ED model are significantly positive, both economically and statistically. The average profit per trade ranges from 1.19 basis point for the 5-day holding period strategy to 49.04 basis points for the 250 -day holding period strategy. The statistical significance of this profit, reported in row 1 of Panel B in Exhibit 8, as measured by the NWstat also exceeds the 5\% level for all 4 holding periods.

Row 3 of Panel A in Exhibit 8 shows that the profitability of the buy-and-hold strategies, while always lower than the CFRS_ED model, are quite similar in terms of magnitude. The buy-and-hold strategy's profits ranges from 1.16 basis point for the 5-day holding period strategy to 48.74 basis points for the 250-day holding period strategy. The closeness between the profitability of these CFRS_ED model and the buy-and-hold is reflective of the fact that the CFRS_ED model will, more often than not, predict a fall in forward rates, due to the fact that unconditionally, the term structure of the forward curve is upward sloping (hence, unconditionally, the drift of forward rates is downwards). The differences in profitability will therefore come from the times when the CRFS_ED model predicts a rise in the forward rates, while the buy-and-hold maintains the downward prediction. Hence, to test the statistical significance between the CFRS_ED model and the buy-and-hold model, we exclude all the dates when the trade signals are identical for both strategies, and only use those dates when their forecasted directions diverge.

Row 2 of Panel B of Exhibit 8 shows that the profitability of the CFRS_ED model is significantly higher than that for the buy-and-hold for all holding periods, and especially so for the 65-day and 250-day holding periods, with NW-stat reaching 7.953.

\section{**** EXHIBIT 8 AROUND HERE ****}




\section{CONCLUSION}

We have estimated and tested a dynamic model for the forward Eurodollar rates. The model has several desirable features, most notably that it

- Captures maturity-specific preferences that arise from preferred habitats,

- Accommodates date-specific expectations about future spot rates,

- Permits estimation and extrapolation via underlying state variables within the framework of a Kalman filter, and

- Has a general structure that permits the choice of a particular model parsimonious in parameters and state variables, while still remaining arbitrage-free.

The selected representative of our model class performs well in forecasts over various horizons, relative to the Random Walk model. The chosen model also supports the construction of a trading strategy that significantly outperforms the returns of a buy-and-hold strategy, especially over longer holding periods.

We leave to future work the task of searching for the superior models from this general class as well as the extension to other types of data. 


\section{EXHIBIT 1: Illustration of Maturity-Specific Deviation Behavior}

Starting with any given maturity-specific deviation (for illustrative purposes, we set the original maturityspecific deviation to be $M(\tau ; t)=0.05 e^{-0.2 \tau}-0.1 e^{-0.4 \tau}+0.05 e^{-0.8 \tau}$. We expect the maturity-specific deviation to decay exponentially to zero at rate $K_{m}$ (In this illustration, we set $K_{m}=0.4$ ) as time passes (from Equation (3)): $E_{t}[M(\tau ; T)]=e^{-K_{m}(T-t)} M(\tau ; t)$.

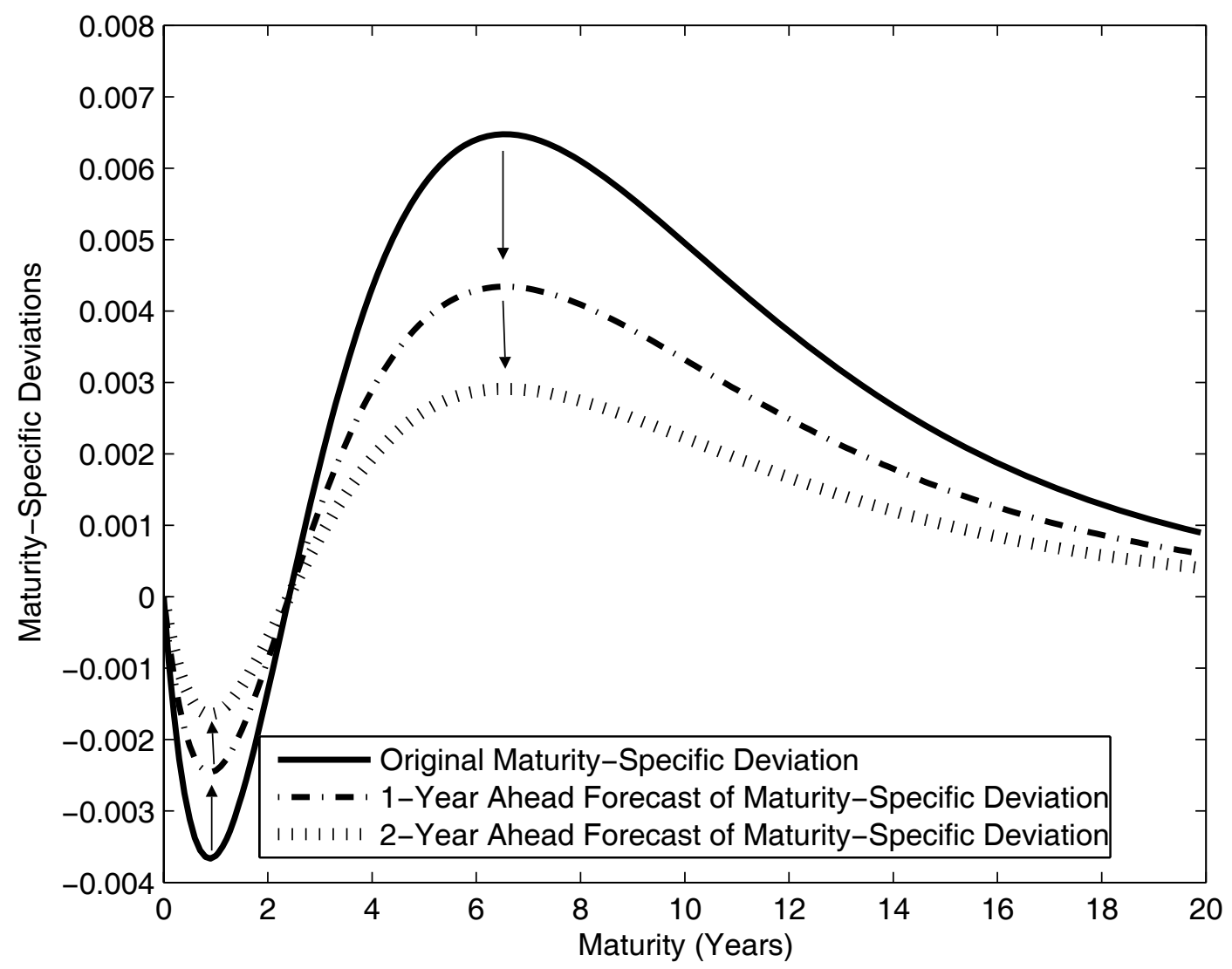




\section{EXHIBIT 2: Illustration of Date-specific Deviation Behavior}

Starting with any given date-specific deviation (for illustrative purposes, we set the original date-specific deviation to be $D(\tau ; t)=0.04 e^{-0.2 \tau}-0.05 e^{-0.4 \tau}$. We expect the date-specific deviation curve to shift to the left uniformly as time passes (from Equation (5)): $E_{t}[D(\tau-(T-t) ; T)]=D(\tau ; t)$.

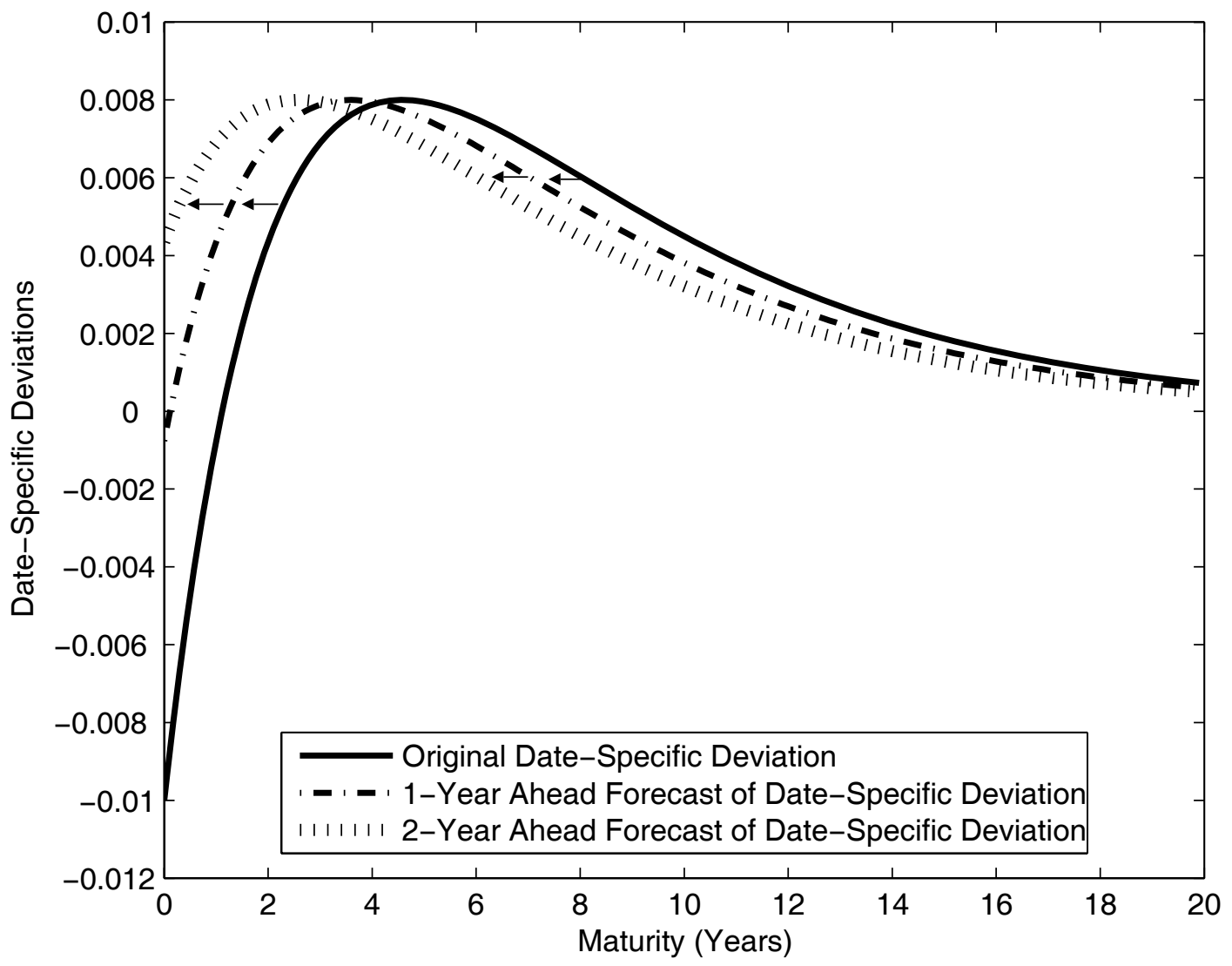


EXHIBIT 3: Summary statistics of Eurodollar futures data for full sample (12/09/1981 to 1/28/2008) and training sample $(12 / 09 / 1981$ to $10 / 28 / 1991)$

We convert Eurodollar futures prices into estimates of instantaneous forward rates via the formula: $f(\tau+$ $45 ; t)=100-P(\tau ; t)$. We report the counts, means and standard deviations of the maturities as well as the forward rates for both the full sample and the training sample. Contract Number refers to the $\mathrm{n}$-th nearest contract to maturity on any particular day.

\begin{tabular}{|c|c|c|c|c|c|c|c|c|c|c|}
\hline \multirow{3}{*}{$\begin{array}{l}\text { Contract } \\
\text { Number }\end{array}$} & \multicolumn{5}{|c|}{ Full Sample } & \multicolumn{5}{|c|}{ Training Sample } \\
\hline & \multirow[b]{2}{*}{ Count } & \multicolumn{2}{|c|}{ Maturity } & \multicolumn{2}{|c|}{ Forward Rates } & \multirow[b]{2}{*}{ Count } & \multicolumn{2}{|c|}{ Maturity } & \multicolumn{2}{|c|}{ Forward Rates } \\
\hline & & Mean & S.D. & Mean & S.D. & & Mean & S.D. & Mean & S.D. \\
\hline 1 & 6600 & 76.7 & 25.6 & 6.091 & 2.939 & 2500 & 91.1 & 26.4 & 8.933 & 2.296 \\
\hline 2 & 6600 & 140.1 & 66.4 & 6.218 & 3.016 & 2500 & 182.4 & 26.5 & 9.140 & 2.379 \\
\hline 3 & 6598 & 203.0 & 79.2 & 6.349 & 3.063 & 2500 & 273.8 & 26.5 & 9.338 & 2.391 \\
\hline 4 & 6541 & 269.0 & 105.8 & 6.410 & 2.998 & 2443 & 364.6 & 26.5 & 9.408 & 2.209 \\
\hline 5 & 6176 & 332.2 & 131.0 & 6.216 & 2.721 & 2078 & 455.5 & 26.4 & 9.138 & 1.825 \\
\hline 6 & 6164 & 402.7 & 155.9 & 6.350 & 2.752 & 2067 & 546.5 & 26.4 & 9.328 & 1.814 \\
\hline 7 & 5907 & 476.9 & 170.7 & 6.223 & 2.465 & 1812 & 638.2 & 26.5 & 9.037 & 1.327 \\
\hline 8 & 5866 & 566.5 & 171.7 & 6.332 & 2.383 & 1774 & 729.2 & 26.4 & 9.116 & 1.211 \\
\hline 9 & 5197 & 637.0 & 172.1 & 6.056 & 2.060 & 1106 & 821.1 & 26.5 & 8.959 & 0.540 \\
\hline 10 & 5196 & 728.4 & 172.9 & 6.184 & 1.994 & 1105 & 912.1 & 26.4 & 9.038 & 0.524 \\
\hline 11 & 5194 & 819.1 & 168.4 & 6.300 & 1.938 & 1104 & 1003.1 & 26.4 & 9.105 & 0.521 \\
\hline 12 & 5188 & 910.1 & 168.2 & 6.400 & 1.892 & 1100 & 1094.1 & 26.5 & 9.166 & 0.513 \\
\hline 13 & 4688 & 981.8 & 164.8 & 6.158 & 1.630 & 600 & 1184.6 & 26.8 & 8.911 & 0.345 \\
\hline 14 & 4685 & 1074.0 & 166.3 & 6.241 & 1.596 & 597 & 1283.8 & 37.9 & 8.957 & 0.333 \\
\hline 15 & 4635 & 1162.0 & 164.2 & 6.295 & 1.558 & 547 & 1367.0 & 27.1 & 9.024 & 0.339 \\
\hline 16 & 4624 & 1252.7 & 164.1 & 6.361 & 1.529 & 536 & 1457.9 & 26.7 & 9.063 & 0.322 \\
\hline 17 & 3924 & 1307.3 & 150.7 & 5.983 & 1.155 & 0 & & & & \\
\hline 18 & 3924 & 1398.4 & 150.7 & 6.053 & 1.137 & 0 & & & & \\
\hline . & . & . & . & & . & . & & & & \\
\hline . & . & . & . & & . & . & & & & \\
\hline 42 & 2924 & 3513.8 & 80.1 & 6.736 & 0.827 & 0 & & & & \\
\hline 43 & 2251 & 3566.6 & 39.6 & 6.500 & 0.719 & 0 & & & & \\
\hline 44 & 1954 & 3648.6 & 30.9 & 6.416 & 0.653 & 0 & & & & \\
\hline 45 & 57 & 3651.9 & 15.0 & 6.406 & 0.555 & 0 & & & & \\
\hline
\end{tabular}




\section{EXHIBIT 4: Log-likelihood and Akaike Information Criterion Values (AIC) in Model Selection using daily Eurodollar futures data from 12/09/1981 to 10/28/1991}

We use the Eurodollar Futures training data to derive the log-likelihood and AIC values for six competing models. The model with the highest log-likelihood and lowest AIC value is our model of choice (CFRS_ED model). AIC is calculated via the formula: $-2 \ln L+2 K$, where $L$ is the likelihood, and $K$ is the number of free parameters in the model. The models that we consider are made up of different combinations of the following 3 sub-models (where $m_{i}(t)$ refers to the maturity-specific state variable of the $i$-th sub-model, and $d_{j, k}(t)$ refers to the $k$-th date-specific state variable of the $j$-th sub-model):

1. Sub-model 1 (SM1), 1 Brownian Motion:

$U(s-t)+m_{1}(t)\left(e^{-K_{m}(s-t)}-e^{-2 K_{m}(s-t)}\right)+d_{1,1}(t) e^{-2 K_{m}(s-t)}$

2. Sub-model 2 (SM2), 2 Brownian Motions:

$U(s-t)+m_{2}(t)\left(2 e^{-K_{m}(s-t)}-2 e^{-2 K_{m}(s-t)}\right)+2 d_{2,1}(t) e^{-2 K_{m}(s-t)}+d_{2,2}(t) e^{-K_{m}(s-t)}$

3. Sub-model 3 (SM3), 2 Brownian Motions:

$U(s-t)+m_{3}(t)\left(2 e^{-K_{m}(s-t)}-e^{-2 K_{m}(s-t)}-e^{-4 K_{m}(s-t)}\right)+d_{3,1}(t) e^{-2 K_{m}(s-t)}+d_{3,2}(t) e^{-4 K_{m}(s-t)}+d_{3,3}(t) e^{-2 K_{m}(s-t)}$

\begin{tabular}{cccccc}
\hline Model & Composition & State Dimensions & Free Parameters & Log-likelihood & AIC \\
\hline Model 1 & SM1 & 2 & $3\left\{C_{0}, C_{1}, K_{m}\right\}$ & $-3.2300 \mathrm{e} 7$ & $6.4600 \mathrm{e} 7$ \\
Model 2 & SM2 & 3 & $3\left\{C_{0}, C_{1}, K_{m}\right\}$ & $-0.4099 \mathrm{e} 7$ & $0.8199 \mathrm{e} 7$ \\
Model 3 & SM3 & 4 & $4\left\{C_{0}, C_{1}, C_{2}, K_{m}\right\}$ & $-0.0985 \mathrm{e} 7$ & $0.1971 \mathrm{e} 7$ \\
Model 4 & SM1 + SM2 & 5 & $3\left\{C_{0}, C_{1}, K_{m}\right\}$ & $-0.8926 \mathrm{e} 7$ & $1.7853 \mathrm{e} 7$ \\
Model 5 & $\mathrm{SM} 1+\mathrm{SM} 3$ & 6 & $4\left\{C_{0}, C_{1}, C_{2}, K_{m}\right\}$ & $-0.0599 \mathrm{e} 7$ & $0.1198 \mathrm{e} 7$ \\
Model 6 & $\mathrm{SM} 2+\mathrm{SM} 3$ & 7 & $4\left\{C_{0}, C_{1}, C_{2}, K_{m}\right\}$ & $-1.3412 \mathrm{e} 7$ & $2.6824 \mathrm{e} 7$ \\
\hline
\end{tabular}




\section{EXHIBIT 5: Illustration of Market Micro-structure Issues}

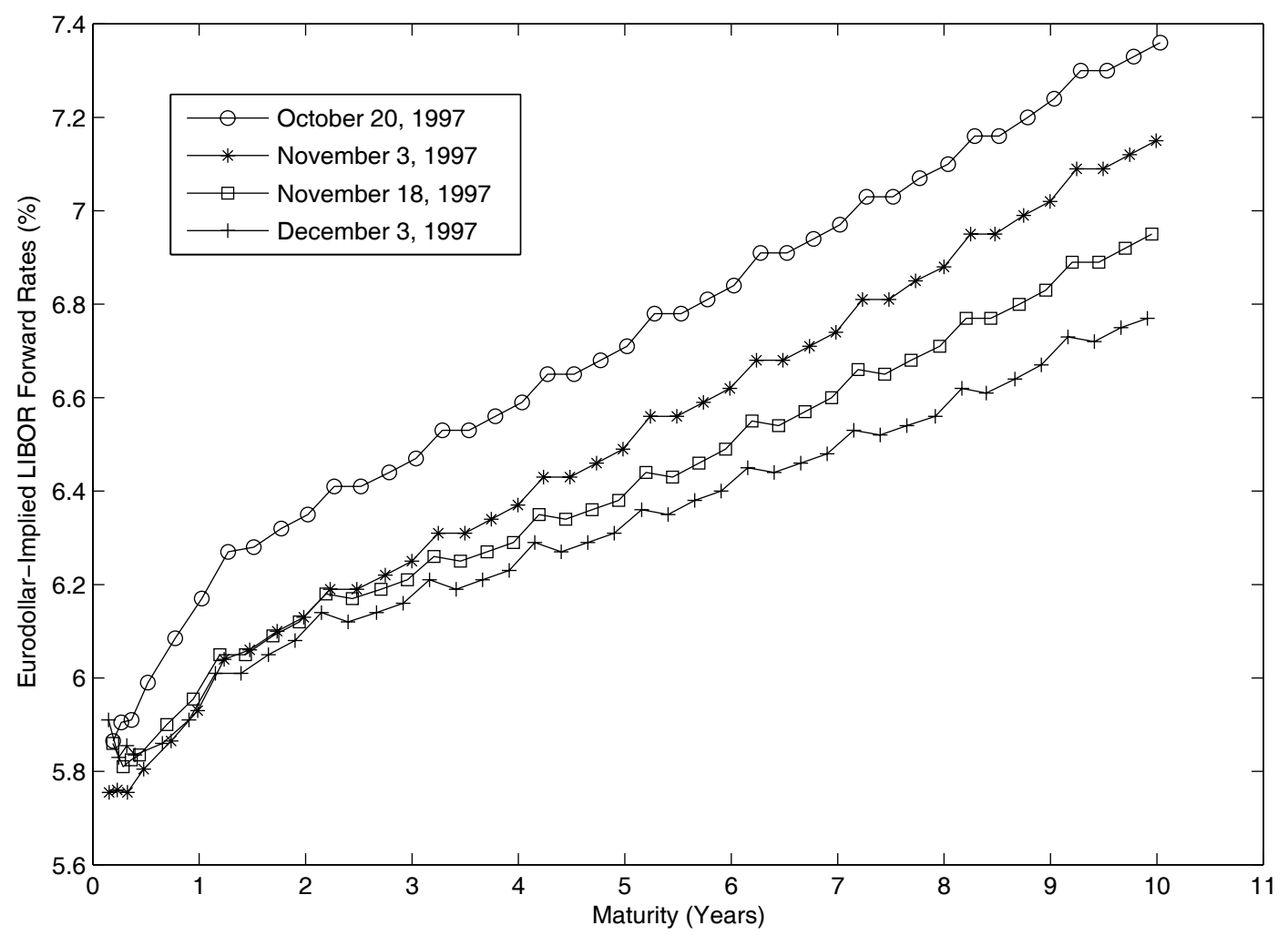


EXHIBIT 6: Timeline for Model Estimation and Forecasts

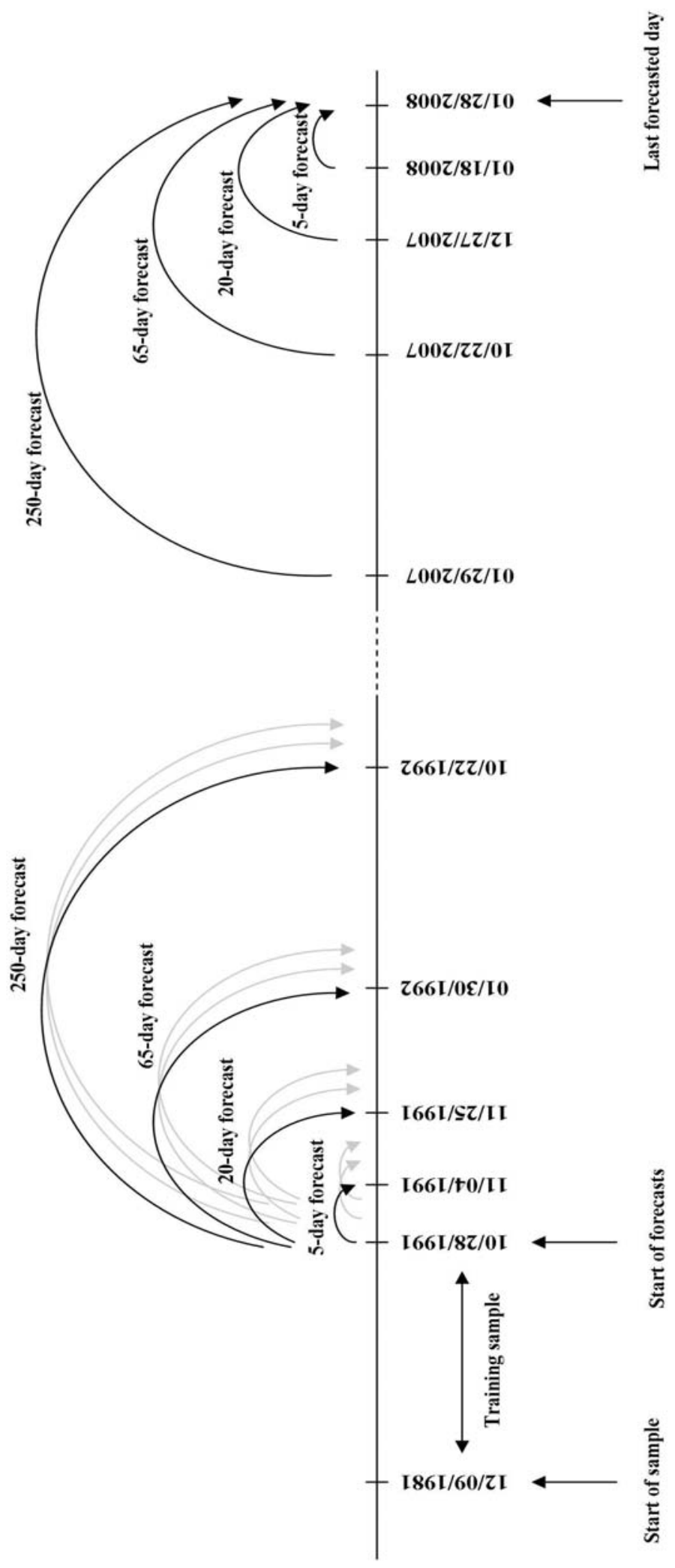




\section{EXHIBIT 7: Accuracy of Out-of-Sample Forward Rate Forecasts}

This table shows the RMSEs of forecasting error of 5-, 20-, 65- and 250-day-ahead forward rate forecasts produced by the CFRS_ED model, and the Random Walk model (RW model). We use the first 2500 days of training data to generate the first forecast. The training window is then expanded forward one day at a time to generate successive forecasts. We first calculate the cross-sectional RMSE of forecast error in any given day. We report the time-series means and standard deviations of these cross-sectional RMSEs in this table. The NW-stat (quoted as a z-score) is used to test the significance of the differences in RMSEs between any 2 models. A negative value of the NW-stat indicates that the first model (the model mentioned before "vs") is performing better than the second model.

\begin{tabular}{|c|c|c|c|c|}
\hline & $\begin{array}{c}\text { 5-day-ahead } \\
(\approx 1 \text { calendar week })\end{array}$ & $\begin{array}{c}\text { 20-day-ahead } \\
(\approx 1 \text { calendar month })\end{array}$ & $\begin{array}{c}\text { 65-day-ahead } \\
(\approx 1 \text { calendar quarter })\end{array}$ & $\begin{array}{c}\text { 250-day-ahead } \\
(\approx 1 \text { calendar year })\end{array}$ \\
\hline Number of Forecasts & 4095 & 4080 & 4035 & 3850 \\
\hline \multicolumn{5}{|c|}{ Panel A: Mean RMSE; standard deviation in parenthesis (in basis points) } \\
\hline CFRS_ED & $\begin{array}{l}12.53 \\
(8.47)\end{array}$ & $\begin{array}{c}25.39 \\
(16.25)\end{array}$ & $\begin{array}{c}48.03 \\
(28.80)\end{array}$ & $\begin{array}{c}98.58 \\
(53.93)\end{array}$ \\
\hline RW & $\begin{array}{l}12.55 \\
(8.51)\end{array}$ & $\begin{array}{c}25.69 \\
(16.36)\end{array}$ & $\begin{array}{c}48.63 \\
(31.46)\end{array}$ & $\begin{array}{l}104.89 \\
(66.64)\end{array}$ \\
\hline \multicolumn{5}{|l|}{ Panel B: NW-stat } \\
\hline CFRS_ED vs RW & -0.594 & -1.030 & -0.365 & -0.658 \\
\hline
\end{tabular}




\section{EXHIBIT 8: Profitability of Trading Strategy}

This table shows the profitability of trading strategies that capitalizes on the forecasts of the CFRS_ED model, as well as a buy-and-hold model. We consider trading strategies over a 5-, 20-, 65- and 250-day holding period. We use the first 2500 days of training data to generate the first forecast. The training window is then expanded forward one day at a time to generate successive forecasts. If the forecasted forward rate is lower (higher) than the current corresponding forward rate, the strategy is to bet that the forward rate will fall (rise) over the forecasted period. Trades are placed for all Eurodollar futures contracts that will still exist at the end of the forecasting period. The profitability on each trade is then calculated as the cross-sectional average movement in (or against) the predicted direction over the length of the forecasting period, expressed in basis points. We report the time-series means and standard deviations of the cross-sectional average. The NW-stat (quoted as a z-score) is used to test the significance of the differences in profitability between any 2 trading strategies, using only days when the trades of the 2 strategies are different. A positive value of the NW-stat indicates that the first strategy (the model mentioned before "vs") is performing better than the second strategy.

\begin{tabular}{|c|c|c|c|c|}
\hline & $\begin{array}{l}\text { 5-day holding period } \\
(\approx 1 \text { calendar week })\end{array}$ & $\begin{array}{l}\text { 20-day holding period } \\
(\approx 1 \text { calendar month })\end{array}$ & $\begin{array}{l}\text { 65-day holding period } \\
(\approx 1 \text { calendar quarter })\end{array}$ & $\begin{array}{l}\text { 250-day holding period } \\
\quad(\approx 1 \text { calendar year })\end{array}$ \\
\hline Number of Forecasts & 4095 & 4080 & 4035 & 3850 \\
\hline \multicolumn{5}{|c|}{ Panel A: Mean Profitability; standard deviation in parenthesis (in basis points) } \\
\hline \multirow[t]{2}{*}{ CFRS_ED } & 1.19 & 4.59 & 14.18 & 49.04 \\
\hline & $(13.70)$ & $(27.25)$ & $(51.42)$ & $(105.48)$ \\
\hline \multirow[t]{2}{*}{ Buy-and-Hold } & 1.16 & 4.46 & 13.75 & 48.74 \\
\hline & $(13.80)$ & $(27.56)$ & $(52.32)$ & $(106.21)$ \\
\hline \multicolumn{5}{|l|}{ Panel B: NW-stat } \\
\hline CFRS_ED vs No Trade & 3.061 & 2.896 & 2.666 & 2.409 \\
\hline CFRS_ED vs Buy-and-Hold & 1.919 & 1.804 & 2.243 & 7.953 \\
\hline
\end{tabular}




\section{APPENDIX}

\section{Checking the HJM Restriction}

We must now verify that the proposed dynamics in Equation (12) is arbitrage-free. Denoting the diffusion of the forward rate SDE as:

$$
\sigma(t, s)=e^{-K_{m}(s-t)} \gamma_{t}, \tau \equiv s-t
$$

we have

$$
\int_{t}^{s} \sigma(t, v) d v=-\frac{1}{K_{m}} e^{-K_{m}(s-t)} \gamma_{t}+\frac{1}{K_{m}} \gamma_{t}
$$

For this version of the 1-Brownian motion arbitrage-free unit, we choose the market price of risk $\kappa_{t}$ as: ${ }^{7}$

$$
\kappa_{t}=\frac{\gamma_{t}}{K_{m}}
$$

Notice that the market price of risk is proportional to the diffusion term of the state variable, just as in the CIR model. Then the HJM condition says

$$
\sigma(t, s)\left(\int_{t}^{s} \sigma(t, v) d v-\kappa_{t}\right)=-\frac{1}{K_{m}} \gamma_{t}^{2} e^{-2 K_{m}(s-t)}
$$

By specifying $\gamma_{t}^{2}$ as:

$$
\gamma_{t}^{2}=\left(m(t)+2 C_{1}\right) K_{m}^{2}
$$

Equation (19) becomes 
$\sigma(t, s)\left(\int_{t}^{s} \sigma(t, v) d v-\kappa_{t}\right)=-K_{m}\left(2 C_{1}+m(t)\right) e^{-2 K_{m}(s-t)}$

which is exactly the drift of $\mathrm{d} f_{1}(s-t ; t)$ (see Equation (12)), thus satisfying the HJM condition.

\section{Fitting the Kalman Filter}

A standard Kalman filter can be used to estimate a system of unobserved state variables in which the observed variables are linked to the unobserved state variables via a measurement equation, and the transition equation for the unobserved state variables is specified as a system of linear equations with Gaussian innovations (see Hamilton [1994] Chapter 13 for a discussion of the Kalman filter's implementation and estimation). If the innovations in the unobserved state variables are not Gaussian (which is the case for our model), estimates from the standard Kalman filter are, in general, not conditionally unbiased estimators of the true state variables (Chen and Scott [2002]). However, it is still possible to proceed with the implementation of the Kalman filter by assuming that the innovations are indeed Gaussian in order to obtain a quasi-log-likelihood from the Kalman filter, and then optimize over that quasi-log-likelihood to obtain quasi-maximum likelihood (QML) estimates for parameters of the model. The parameters in the model that we need to optimize over the quasi-log-likelihood are $K_{m}$, the decay rate of the maturity-specific deviation, and $\sigma^{* 2}$, the variance of the measurement errors.

By viewing $\vec{m}(t)$ and $\vec{d}(t)$ as latent state variables we are able to fit our model directly into a Kalman filter framework. Stack a sequence of maturities into a vector $\vec{\tau}=\left[\tau_{1}, \ldots, \tau_{\ell}\right]^{\prime}$. Next place $\vec{m}(t)$ and $\vec{d}(t)$ into a vector $\vec{x}_{t}$ :

$$
\vec{x}_{t}=\left[\begin{array}{c}
\vec{m}(t) \\
\vec{d}(t)
\end{array}\right]
$$

At each date $t$, we can relate these to the observed data with the measurement equation:

$$
\vec{z}_{t} \equiv f(\vec{\tau} ; t)-\hat{U}(\vec{\tau})=\mathbf{A} \vec{x}_{t}+\vec{\varepsilon}_{t}
$$

where $\mathbf{A}$ is the measurement matrix for the state variables, and $\vec{\varepsilon}_{t}$ is the vector of measurement errors. The $j$-th row of the matrix $\mathbf{A}$ is defined as 


$$
\left.\mathbf{A}_{j} \equiv\left[\left(e^{\vec{k} \tau} \tau_{j}\right)^{\prime} \mathbf{M} ;\left(e^{\vec{k} \tau}\right)_{j}\right)^{\prime} \mathbf{D}\right]
$$

To allow for statistical estimation, we now simplify the model by adding the assumption that the measurement errors are homoscedastic and both cross-sectionally and serially uncorrelated:

$$
\Sigma_{\varepsilon} \equiv \operatorname{Var}\left(\vec{\varepsilon}_{t}\right)=\sigma^{* 2} I
$$

We estimate the noise variance $\sigma^{* 2}$ from the data when maximizing the quasi-likelihood.

We can now derive the transition equation of the Kalman filter as the discretized version of the stochastic process for $\vec{x}_{t}$. First let

$$
\mathbf{V}=\left[\begin{array}{c}
\mathbf{V}_{m} 0 \\
0 \mathbf{V}_{d}
\end{array}\right] \text { and } \Sigma(\vec{m})=\left[\begin{array}{c}
\Sigma_{m}(\vec{m}) \\
\Sigma_{d}(\vec{m})
\end{array}\right]
$$

The transition equation is therefore

$$
\vec{x}_{t}=W \vec{x}_{t-1}+\xi_{t}
$$

where $W$ is a diagonal matrix with

$$
W_{i i}=e^{\delta \mathbf{V}_{i i}}
$$

where $\delta$ is the step size, and we approximate $Q_{t} \equiv \operatorname{Var}_{t-1}\left(\xi_{t}\right)$ by

$$
Q_{t} \approx \delta \Sigma(\vec{m}) \Sigma(\vec{m})^{\prime}
$$

Given this specification for the Kalman filter, we set the initial estimates of the state vector at its unconditional mean, which is zero $\left(\hat{\vec{x}}_{0}=0\right)$, and set the initial covariance matrix at the unconditional variance $\operatorname{Var}\left(\vec{x}_{t}\right)$. We can then run the Kalman filter to estimate the state variables by iterating between the prediction equations and the updating equations as in DeJong and Santa-Clara [1999], Geyer and Pichler [1999] and Babbs and Nowman [1999]:

The predicting equations: 
$x_{t \mid t-1}=W \hat{x}_{t-1 \mid t-1}$

where $x_{t \mid t-1}$ is the time $t-1$ prediction of $x_{t}$ and $\hat{x}_{t-1 \mid t-1}$ is the time $t-1$ estimate of $x_{t-1}$, and

$P_{t \mid t-1}=W \hat{P}_{t-1 \mid t-1} W^{\prime}+Q_{t}$

where $P_{t \mid t-1}$ is the time $t-1$ prediction of $P_{t}$ and $\hat{P}_{t-1 \mid t-1}$ is the time $t-1$ estimate of $P_{t-1}(P$ is the covariance matrix of the state vector $x$ ).

Updating equations:

$\hat{x}_{t \mid t}=x_{t \mid t-1}+P_{t \mid t-1} A^{\prime} H_{t}^{-1} v_{t}$

$\hat{P}_{t \mid t}=P_{t \mid t-1}-P_{t \mid t-1} A^{\prime} H_{t}^{-1} A P_{t \mid t-1}$

where

$v_{t}=z_{t}-A x_{t \mid t-1}$

are the prediction errors, and

$H_{t}=A P_{t \mid t-1} A^{\prime}+\sigma^{* 2} I$

is the conditional variance of the prediction errors.

The log-likelihood function is then: $\ln L=-\frac{n T}{2} \ln 2 \pi-\frac{1}{2} \sum_{t=1}^{T}\left(\ln \left|H_{t}\right|+v_{t}^{\prime} H_{t}^{-1} v_{t}\right)$ 


\section{REFERENCES}

Akaike, H. "Information Theory and an Extension of the Maximum Likelihood Principle." Second International Symposium on Information Theory, (1973), 267-281.

Babbs, S., and K. Nowman. "Kalman Filtering of Generalized Vasicek Term Structure Models." Journal of Financial and Quantitative Analysis, 34 (1999), 115-130.

Chen, R., and L. Scott. "Multi-factor Cox-Ingersoll-Ross Models of the Term Structure: Estimates and Tests from a Kalman Filter Model.” Working Paper, Rutgers University, 2002.

Chua, C., D. Foster, K. Ramaswamy, and R. Stine. "A Dynamic Model for the Forward Curve.” Review of Financial Studies, 21 (2008), 265-310.

Cox, J., J. Ingersoll, and S. Ross. "A Re-examination of Traditional Hypotheses about the Term Structure of Interest Rates." Journal of Finance, 36 (1981), 769-799.

—. "A theory of the Term Structure of Interest Rates." Econometrica, 53 (1985), 385-407.

DeJong, F., and P. Santa-Clara. "The Dynamics of the Forward Interest Rate Curve: a Formulation with State Variables.” Journal of Financial and Quantitative Analysis, 31 (1999), 131-157.

Diebold, F., and R. Mariano. "Comparing Predictive Accuracy." Journal of Business and Economic Statistics, 13 (1995), 253-263.

Fisher, I. “Appreciation and Interest." Publications of the American Economic Association, 11 (1986), 2129.

Fisher, M., and C. Gilles. "Around and Around: the Expectations Hypothesis." Journal of Finance, 53 (1998), 365-383.

Geyer, A., and S. Pichler. "A State-space Approach to Estimate and Test Multifactor Cox-Ingersoll-Ross Models of the Term Structure.” Journal of Financial Research, 22 (1999), 107-130. 
Hamilton, J. Time Series Analysis. Princeton, NJ: Princeton University Press, 1994.

Longstaff, F. “Arbitrage and the Expectations Hypothesis.” Journal of Finance, 55 (2000), 989-994.

McCulloch, J. "A Re-examination of Traditional Hypotheses about the Term Structure: a Comment." Journal of Finance, 48 (1993), 779-789.

Modigliani, F., and R. Sutch. "Innovations in Interest Rate Policy." The American Economic Review, 56 (1966), 178-197.

Newey, W., and K. West. "A Simple, Positive Semi-definite, Heteroskedascity and Autocorrelation Consistent Covariance Matrix." Econometrica, 55 (1987), 703-708.

Vasicek, O. "An Equilibrium Characterization of the Term Structure." Journal of Financial Economics, 5 (1977), 177-188. 


\section{ENDNOTES}

${ }^{1}$ The CFRS model does not account for default and (strictly speaking) applies to forward rates implicit in Treasury yields. The Eurodollar futures market settles to the rate on dollar-denominated inter-bank loans in London and therefore is affected by a credit risk; we choose it because is a very liquid market and offers a rich source of data.

${ }^{2}$ Some recent literature seem to vindicate theoretical aspects of the Expectations Hypothesis. McCulloch [1993] and Fisher and Gilles [1998] present examples to show that some forms of the Expectations Hypothesis are consistent with no-arbitrage. Longstaff [2000] shows that all traditional forms of the Expectations Hypothesis are consistent with no-arbitrage if markets are incomplete.

${ }^{3}$ See CFRS [2008] for further discussion of the connection of their model to other models.

${ }^{4}$ The instantaneous forward rate on date $t$ for maturity on date $s$ is really a function of $\{t, s-t, m(t)$, and $d(t)\}$ where the final two arguments are state variables that affect the maturity- and date-specific deviations. For simplicity, we will continue to write our model for the forward rate as a function of 2 variables: $\{\tau=s-t, t\}$, writing $F_{1}(\tau ; t)$ in place of $F_{1}(t, s, m(t), d(t))$ and suppressing the dependence on the two state variables.

${ }^{5}$ The precise choice of exponential bases can affect the arbitrage-free status of the model, making it important to verify the HJM conditions for each choice.

${ }^{6} \mathrm{We}$ first compute the difference between a given day's cross-sectional RMSE for our model and the RW model. Then, we compute the Newey-West (NW) standard error for the time-series of these differences. The mean difference divided by this NW standard error is our reported NW statistic. Alternatively, we can compute the significance using the Diebold-Mariano [1995] method.

${ }^{7}$ It is not necessary that $\kappa_{t}=\frac{\gamma_{t}}{K_{m}}$. If the market price of risk takes on another form, the model requires a different specification for $\gamma_{t}$ or $U(s-t)$ or both so that the system remains arbitrage-free.

${ }^{8}$ The framework of the model places boundaries on the values of some of the state variables. The diffusion terms, which are functions of the maturity-specific state variables, must be constrained to be non-negative. This in turn places constraints on those state variables. In the empirical implementation, a simple and common way of enforcing this restriction is to replace the values of the state variables that do breach the constraints with ones that just satisfy it. See Chen and Scott [2002] and Geyer and Pichler [1999] for further examples of such restrictions in a Kalman filter. 